\title{
Modeling dynamical function: sparsely synchronized frameworks for deep brain stimulation?
}

\author{
Denis Larrivee \\ Loyola University Chicago, USA and Mind and Brain Institute, University of Navarra, Pamplona, Spain
}

\begin{abstract}
High frequency electrical stimulation of deep brain structures, known as deep brain stimulation (DBS), has been successfully used to treat a number of refractory neurological, movement, and psychiatric related, disorders; yet its mechanism of action is largely unknown. Employed for most movement neuropathologies, the expanding number of therapeutic applications suggests a mechanism of action mimicking basic brain operation, oscillatory activity. A general hypothesis for its effectiveness is that DBS ameliorates cognitive dysfunction through a generalized disruption of aberrant oscillations, such as the heightened $\beta$ oscillations seen in Parkinson's Disease (PD). Existing evidence suggests, however, that its mechanism of operation is likely to be more complex and may be better framed as an interaction with sparsely synchronized frameworks for neural transmission. .
\end{abstract}

\section{Introduction}

High frequency electrical stimulation of deep brain structures, known as deep brain stimulation (DBS), has been successfully used to treat a number of refractory neurological, movement and psychiatric related, disorders, yet its mechanism of action is largely unknown. In movement disorders DBS is therapeutic for tremors, dystonia, and dyskinesia, yielding a 50\% improvement in PD on the Unified Parkinson's Disease Rating Scale [1]. In psychiatric diseases DBS shows promise for treatment of obsessive compulsive disorder and Gilles de la Tourette syndrome, and has a limited impact on major depressive syndrome.

Historically, high frequency electrical stimulation came into practice as a therapeutic intervention to replace ablative surgery for Parkinson's in the mid-20th century, although electric stimulation had been used in treatment of nervous system disorders for centuries before [2]. Thalamotomies and pallidotomies successfully abolished the tremors, but their permanent effects were deemed unsuitable when it was observed that the effects of DBS were not only similar to those introduced by surgery but were also reversible; potentially adverse side effects of DBS, thus, were removed when the stimulus was terminated. From its initial use for Parkinson's the spectrum of therapeutic interventions has since greatly broadened, and now includes most movement neuropathologies as well as an increasing number of psychiatric diseases.

The expanding number of therapeutic applications for which DBS is employed suggests that its mechanism of action likely involves basic operational features used by the brain. Because the form of stimulation is rhythmic there is the implicit likelihood that these corresponding neural mechanisms are similarly rhythmic; increasingly, these are proposed to be oscillatory [3]. Rhythmic activity, notably, is a ubiquitous feature of brain operation, and has been observed at the single neuron level, with varying spiking patterns [4], as well as at network levels, with variable inhibitory and excitatory feedback configurations [5]. Its elicitation and propagation appear to be due to such factors as subthreshold currents, originating from post synaptic potentials and rhythmic firing of synchronized neural groups [6], as well as physical properties like anatomical configuration and noise contributions [7], that together yield a complex resonating architecture.

The significance of oscillatory activity in brain function was first made apparent through its ability to address a cardinal difficulty of the hierarchical abstractive model, proposed by Wiesel and Hubel [8], and used to explain how information was processed in the visual cortex. In this model input from the retinal ganglion cells was integrated through successive neuronal layers to assimilate an increasingly complex and abstract portrait of sensory information. Cortical motion detector cells, for example, determined stimulus speed by summating inputs from sequentially stimulated, retinal photoreceptors, and orientation cells then integrated the summated inputs to ascertain the stimulus' direction. The hypothesized abstraction, however, had the conceptual consequence of progressively restricting the numbers of neurons that participated in the abstraction, eventually limiting them to a small population of cardinal neurons stated to contain the salient sensory information. Oscillators, by contrast, can combine in virtually any combination to recreate encoded feature representations. Hence, in the oscillation model there is no numerical neural restriction with information regress and so, potentially, an infinite number of combinatorial variants can be generated $[6,9]$.

How DBS may ameliorate disease conditions thus plausibly entails modulation of oscillatory activity, presumably by modifying intrinsic features of oscillations, like maintenance, synchronization and desynchronization. Indeed, current thinking favours etiological disease models where aberrant oscillatory functioning is directly linked to the observed symptoms and where DBS could intervene by modifying

Correspondence to: Denis Larrivee, Loyola University Chicago, USA and Mind and Brain Institute, University of Navarra, Pamplona, Spain, E-mail: sallar1@ aol.com

Received: March 15, 2018; Accepted: March 29, 2018; Published: March 31,2018 
oscillation rhythms. Among the prevailing hypotheses on how DBS ameliorates cognitive dysfunctions is through a generalized disruption of aberrant oscillations [10,11] like those that are symptomatic of Parkinson's. Classically, however, DBS effectiveness in Parkinson's has been premised on the mimicking of ablative surgery of the subthalamic nucleus (STN) $[3,12]$. The overactivity of the STN and its target, the globus pallidus, which was postulated to underlie the motor symptoms of Parkinson's, as well as its clinical inactivation by surgery and loss of symptoms, led to the suggestion that DBS suppressed STN output through a modulation of STN firing rates. This view has been challenged by Carlson et al. [10] who find no change in STN firing but, rather, the elicitation of random nerve patterning of STN neurons during stimulation. Based on these contraindications DBS is proposed to suppress abnormal oscillations by randomizing or otherwise interrupting disease induced patterns. Whether this explanation is broadly consilient with current findings and what mechanism, if any, may be invoked for DBS is here discussed in regard to two frequently invoked theoretical models of oscillatory behaviour, the Theory of Weakly Coupled Oscillation (TWCO) [13] and the sparse network model for synchronized communication $[5,14]$. It is proposed that DBS development may better invoke theoretical models of oscillatory activity in therapeutic strategy.

\section{DBS and oscillatory features}

\section{Synchronization and desynchronization}

Intuitively, invoking a single mechanism of action for DBS is appealing, since the technical implementation is not confounded by complex technical outcomes, that is, technical devices yield output more clocklike than biological [5], and the proposed creation of competing interferences to reduce oscillatory power is consilent with physical principal. Yet, whether DBS evokes such extensive disassociation, and the part that may be played by disassociation in ameliorating aberrant oscillations, remains unsettled. Tremors in Parkinson's, for example, persist in the waking state, when motions are most frequent, but which has traditionally been characterized as disassociated - a circumstance likely designed to promote maximal information transfer in neural coding - and are reduced, or at least apparently so, in sleep, when large amplitude slow oscillations are evident [7]. These observations, rather, seem to indicate a more complex influence of DBS on oscillatory behaviour.

The complexity of this influence is seen in the several now known features of neural oscillations and how these underwrite brain operation. Aberrations, introduced, by definition, by disease, can alter these features, modifying oscillatory behaviour and changing the sort of interaction that DBS structures with oscillators in order to relieve the diseased state. Their intrinsic ability to combine, for example, is achieved by the synchronization of their frequencies, that is, they mutually adapt their rhythms, a physical observation made centuries ago by Huygens [13]. Although the mechanisms by which neural synchronization occurs are not precisely known, spike timing appears to be an important factor, though a complex one [5]. Oscillations enhance neuronal spike probability by defining, through repetitive cycles, periods of higher excitability, where neurons are sensitive and more responsive to incoming trains, and periods of reduced sensitivity, where spike occurrence is less. This means that there exist intrinsic tendencies toward synchrony where oscillators can properly align, and their frequencies then resonate in unison. Due to their ubiquity throughout the nervous system they are capable of extraordinary combinatorial variety, including interareal as well as interlevel combination. Neural synchronization in the gamma frequency range, notably, has been reported in cortical and subcortical structures [12]. Their regulation, while unknown in details, is thus undoubtedly multifaceted and likely to be multiply impacted by DBS.

Resonating in unison, termed phase locking, has the important effect of enhancing information transfer (Pierce, 1981), hence of facilitating communication between brain domains. However, in the brain, rigid or full phase locking is, in fact, rarely if ever achieved [13]. Some insight into why oscillation resonance is less than perfectly attained can be ascertained from the role played by oscillators in yielding functionally salient outcomes, not solely through a unimodal synchrony but also through recombination. The basic need to reform multiple combinatorial units that will yield functionally differing outcomes, requires that two oscillators disengage so that their frequencies are no longer aligned, that is, they become desynchronized. For example, both beta and mu basal ganglia rhythms show event related desynchronization prior to movement, with sustained suppression during movement execution [15]. Desynchronization necessarily entails a discretized segregation of the oscillators, that is a qualitative separation of the two that is fundamentally a non-linear and dynamical event [16] for which several mechanisms are proposed, like phase resetting through pulsing, or noise induced effects that entrain localized rhythms. Synchronization and desynchronization thus help the brain to achieve reliability and stability of outcome, as well as the flexibility to attain functional variance. Additionally, these needs must be contextualized in terms of global operation, which ultimately defines the sorts of mechanisms needed for the 'good' of the whole individual; hence, oscillatory behaviour is made complex in satisfaction of organismal demand.

\section{Weak associations}

Due to the intrinsic tendency for oscillators to align (or disengage), due to effects on spike timing, influences of DBS on neural oscillators can be expected to specifically impact the spectral power attained during DBS frequency output, including factors like the extent of phase alignment in the population [17], frequency modulation due to the phase dependency of the coupling constant [13], intermittency of alignment [18], and oscillator disruption that may be occasioned by excessive coupling strength [19]. Significantly, the resident oscillator field is not unimodal, but represents a broad distribution of oscillator attractors elicited by the network connectivity and determined by the physical parameters that give rise to them as, for instance, impedance resonance and anatomical configurations. High frequency input applied here may synchronize with a subset of the field population of oscillators but may do so only if the relative synchronizing tendencies between the DBS stimulation and the neural oscillator are energetically preferred to other oscillator combinations, where local instabilities may otherwise effect disengagement. For example, an important class of oscillator synchronies includes those between globally distributed and regionally entrained ones [20]. How, and whether, DBS can extend its influence to larger oscillator pockets is thus uncertain [21].

Mechanistic inferences about DBS, instead, may need to come from recently developed theoretic proposals, for which there is increasing evidence. The need to retain trajectory options in oscillatory combinations, for instance, necessitates that synchronization be only of modest strength, which, accordingly, has been modelled by the Theory of Weakly Coupled Oscillators, mathematically described by the Adler equation [13]. Weak here means that interactions lead to phase adjustments without strong perturbations of the oscillatory generative mechanisms. The Adler equation, accordingly, includes terms for 
divergence, that is, detuning, which is related to the differences in intrinsic oscillator frequencies, and for merger, which is described by the coupling constant, and which is a sine function of the phase angle difference. Weak coupling, in consequence, arises from the opposing tendencies between these two factors that determine the trajectory undertaken toward synchrony. Because phase locking, understood as the constancy of the instantaneous phase relation, is typically never fully achieved, phase precession between the phase of the high frequency stimulation of DBS and that of the resident oscillator necessarily precesses through all phase angles and the coupling strength is continually changing throughout the phase precession cycle. Attractive pull is enhanced when phase tuning is more proximate and reduced when widely separated, that is, the fraction of cycle time respent in phase proximity is greater than that when separated, a circumstance that is increasingly asymmetric as phase locking values approximate to 1 . Worded otherwise, phase precession is slowest when phase angles are closely aligned and fastest when they are farthest apart.

While this suggests that with suitable modification DBS could adequately track and capture an aberrant oscillation, several authors observe that when coupling constants exceed the Kuromoto value, a point at which the repulsive forces due to intrinsic frequency differences are equal to the attractive tendency to align, oscillators undergo increasingly unstable trajectories subject to interferences and destabilizing influences that can terminate cyclical behavior altogether [19]. Hence, in these circumstances the generative mechanisms for oscillations become sufficiently perturbed to induce misalignment and interference.

Consistent with TWCO, and further complicating interpretations of mass effects on neuronal oscillations [10], is a temporal variability that is normally seen in rhythmic neural activity termed phase variance that has been linked to the individual behaviour of micro-oscillators [18]. Such activity displays considerable 'noise' induced variation that is unlike the high frequency, clocklike, oscillations of DBS. This 'noise' has been shown to be due in part to intraneuronal temporal variation in spike production [17], observed with single neuron, current injection episodes [17], and also to variability between groups of neurons that are linked within the oscillating circuits [21]. Temporal variation observed in a mass cranial recording like the EEG, therefore, is likely to indicate a population of oscillators whose alignment is both individually independent and stochastically influenced. Set members, in consequence, exhibit normally distributed variations of phase around a principal mean phase imposed by the external input.

Significantly, such studies of fine structure of the population also show that members of its set regularly deviate from a simple normal distribution, displaying, rather, intermittent episodes of desynchronization, where they are no longer aligned with the principal oscillating phase. The probability of this desynchronization occurs in inverse relation to its duration, that is, short quick desynchronizations are regularly experienced at random intervals and for random lengths by all set members [18]. A significant question raised by this independence is whether DBS induced oscillator entrainment modulates only a subset of oscillator pockets or whether its application will necessarily require coupling strengths that exceed physiological ranges [11].

\section{DBS effects and sparsely synchronized oscillation}

\section{Contraindications}

Cumulatively, the portrait emerging from these observations, as well as from a general consideration of the highly complex behavioural variation undergirded by neural oscillations, suggests a globally asynchronous and stochastic environment within which multiple oscillation patterns are embedded. This is likely to mean that oscillation patterns in nuclei like the globus pallidus, for example, are sparse and probabilistically determined. Indeed, the long prodromal phase in the case of PD, lasting decades, during which substantial depletion of dopaminergic reserves and disruption of synaptic transmission machinery occurs, suggests that communication coherence does not require dedicated, that is, 'rich', neural networks for synchrony [22], but rather one that entails limited neural resources.

It is plausible, by extension, to infer that disease conditions, like Parkinson's, modify oscillatory states normally structured through sparse synchronization [5], that is, the disease, by definition, is defined in terms of its relation to the sparsely synchronized state. Hence, within a variable range of performance, operational vestiges resembling this framework are retained, that is, oscillatory variation due to disease, still assumes an embedded profile within a larger, relatively stochastic population that is then modified by DBS stimulation.

Among the most intensively investigated cognitive diseases for which DBS has been therapeutically used is Parkinson's, which will be considered here as exemplary. From the vantage of a sparsely synchronized thesis one may ask, accordingly, how its constituent oscillatory pathologies may deviate from such frameworks, that is, whether they do and in what manner, and whether DBS may ameliorate PD's motor symptoms by a direct influence on oscillatory pathologies, such as an interference mechanism of the sort proposed by Carlson et al. [10]; or, conversely, independent of or through some combination of effects over and above the chief oscillatory changes observed. In this vein, it may be noted that at least one potential therapeutic mechanism is the restoration of one or more features of normal oscillations activity; this is to say that DBS may precipitate multiple effects only one of which is recuperative for etiology, and that other apparent effects may be epiphenomenal [12]. This question is of significance both from a philosophy of clinical praxis view, since one may ask whether the therapy ought to only alleviate the perceived symptoms, and is also raised in the specific case of $\mathrm{PD}$, since current evidence is not fully conclusive that its signature pathologies, enhanced $\beta$ oscillations, are wholly responsible for the observed motoric symptoms.

$\beta$-oscillations have traditionally been associated with a preparatory phase for and inhibitory control over movement. $\beta$ power, for example, is decreased at the beginning of movement and increases when a movement is withheld [23]. It is implicated in PD through its especial prominence in the basal ganglia of PD patients where higher and lower frequencies occur and is also seen in the subthalamic nucleus; when treated with dopaminergic medication, moreover, beta power in the lower range of the subthalamic nucleus is greatly dissipated [12,24].

Conversely, $\beta$-oscillations comprise a frequently observed cortical rhythm falling within the intermediate frequency range of brain based oscillations, $15-30 \mathrm{~Hz}$, that lies between gamma, $30-80 \mathrm{~Hz}$, and above theta, $3-8 \mathrm{~Hz}$, and slower, frequencies. Initially observed in the primary cortex by Hans Berger in 1931, $\beta$ oscillations are ubiquitous throughout the cortical and subcortical regions. Oscillations similar in frequency to those of $\beta$, for example, are seen in inner cortical layers and in populations of pyramidal cells [5]. Hence, this universality raises questions with regard to how they may play a primary role in a disease that is highly selective in its localization.

Proposed mechanisms of cortical $\beta$ oscillation, further, are 
not easily reconciled with models of sparse random networks and with intermittency. Typical mechanisms for generating rhythmic behaviour reciprocally combine fast positive feedback with slower negative feedback in dedicated, that is, coupled, circuits. On the other hand, some sparsely connected models of Hodgkin Huxley behavior in pyramidal cells and interneurons appear to yield oscillations both in gamma and very low $\beta$ ranges, suggesting a potential, but limited contribution from this source [25].

Another caveat rests in the oscillatory resonances that motor nuclei share with their cortical counterparts. A wide range of cortical frequencies, for example, are elicited in anticipation of and during voluntary movements [12] and existing evidence indicates that these are distinct both in origin and generation. Further, model studies of the subthalamo-pallical network exhibit multiple resonances in response to cortical $\beta$ input [21], suggesting that intrinsic mechanisms within these nuclei are not exclusively tied to the transmission of cortically determined frequency ranges. Such studies are internally consistent with others distinguishing preferred frequency ranges for differing types of motor responses, like $20 \mathrm{~Hz}$ for grip force slowing and 5 and $10 \mathrm{~Hz}$ for finger tapping [26]. No single oscillation, thus, appears to be responsible for all PD symptoms, and so effects of DBS may not be linked to a single oscillatory effect.

\section{Sparse Synchronization}

How then is DBS acting in PD? Although many features of PD improve with DBS, various symptoms respond differently with respect to one another and with respect to the site and manner of stimulation. For example, there is a $50 \%$ improvement in off related motor symptoms like rest tremor, rigidity, and akinesia [1]. It may, for instance, be an oversimplification to consider oscillatory synchrony within a given frequency band to be functionally significant. Task related changes in synchrony occur across multiple frequency bands, and cross frequency associations have been reported. High frequency stimulation, for example, has been shown to yield wide alterations in induced LFP oscillations in rate suggesting that $b$ oscillations may not be the sole oscillatory phase impacted therapeutically by DBS.

Indeed, DBS has undergone considerable variation in methodological protocol as it has developed empirically in attempts to yield improved therapy, generating competing claims with regard to efficacy; hence, developments in DBS have been largely of empirical origin [11]. Appropriate calibration of stimulation parameters like frequency and intensity, width and shape of pulsing stimulation, and spatial spread and localization of current distribution within the tissue have constituted, for example, some of the variation in application that has been explored. An early approach to DBS, for instance, has been the use of open loop applications, where a permanent high frequency electrical train is administered to target brain areas irrespective of the unique regional dynamics. Due to side effects recent methods have used feedback or closed loops where stimulation parameters are given only in response to the detection of dynamical changes in the target tissue; together with suitable computational modelling to compensate for plasticity effects, designated adaptive or aDBS, statistically improved clinical scores can be obtained [11].

The success of DBS in reducing beta oscillations, nonetheless, has led to an emphasis on desynchronization of oscillatory behaviour; that is, PD synchronization has been viewed as pathological and the principal contribution to disease symptoms. Thus, aDBS has been developed to counteract observed neuronal synchrony by ondemand, controlled, desynchronizing stimulation. Accordingly, spatio temporally patterned desynchronizing techniques have been developed in a framework of models that are specifically premised on a need for desynchronization.

Yet whether DBS activates through synchronization or inactivates through desynchronization of neuronal circuits is unclear, and frequently debated $[27,28]$. High frequency stimulation, for example, induces increased neurotransmitter release in first order neuronal projections [29]. Moreover, spike discharges time locked to the stimulation pulses demonstrate the capacity of DBS to drive neurons at the frequency of stimulation by directly activating their neurons. These observations, instead, suggest an influence mediated through synchronization.

Indeed, regulation of cortical inhibitory access may not fully explain the modulatory role played by dopamine, that DBS may replace, or what is to be inferred from PD's exceptionally prolonged prodromal phase prior to the appearance of symptomatic motor behavior [22]. For example, rather than producing global, nonspecific elevations in firing rate for maze learning tasks, dopamine depletion in rats strongly affects learning-related patterning of fast spiking interneuron groups and striatal projection neurons. Yet, while L-DOPA treatment restores normal activity to projection neurons, fast spiking interneurons are unaffected [30]. Dopamine loss, moreover, appears to affect recollection by diminishing the ability to retrieve encoded information since its replenishment restores normal recall of motor tasks [31].

How dopamine may facilitate coding of cortical rhythms in sparse circumstances is uncertain, but could entail phase coding adjustments that activate new resonances such as those observed between cortical and subcortical zones. With sufficient spatial discrimination [15] information transfer would be limited only to discrete oscillator pockets, a circumstance for which only low levels of transmitter would be needed for coding variability. Hence, unlike the off/on gating proposed for beta oscillation, which may be occasioned through phase interference, encoding the selection and processing of cortical transmission may constitute a second and equally significant function of dopamine. Because the resident oscillator field is not unimodal, but represents a complex distribution of oscillator attractors, DBS input, like that from cortical zones, could synchronize with a subset of the field population of energetically preferred oscillators [20], like the important class of oscillator synchronies engaged between globally distributed and regionally entrained ones.

\section{Conclusion}

Among broader issues raised by the expanding use of DBS is that of accounting for its beneficial effect. To date most studies have approached this question empirically, seeking to optimize application by reducing the severity of disease symptoms, without resolving whether these are its only influences or even epiphenomenal ones. Considerable progress has been made in the understanding of normal dynamical physiology, a platform that allows inferences on how disease states deviate from normal physiology. By extension, similar premises may place DBS on a firmer theoretical footing also.

\section{References}

1. Krack P, Batir A, Van Blercom N, Chabardes S, Fraix V, et al. (2003) Five year follow up of bilateral stimlation of the subthalamic nucleus in advanced Parkinson's disease. N Engl J Med 349:1925-1934. [Crossref]

2. Schwalb JM, Hamani C (2008) The history and future of deep brain stimulation. Neurotherapeutics 5:3-13. [Crossref] 
3. Udupa K, Chen R (2015) The mechanisms of action of deep brain stimulation and ideas for the future development. Prog Neurobiol 133: 27-49. [Crossref]

4. Izhikevich EM (2007) Dynamical Systems in Neuroscience: The Geometry of Excitability and Bursting. London: MIT Press.

5. Wang XJ (2010) Neurophysiological and computational principles of cortical rhythms in cognition. Physiol Rev 90: 1195-1268. [Crossref]

6. Kuhn AA, Trottenberg T, Kivi A, Kupsch A, Schneider GH, et al. (2005) The relationship between local field potential and neuronal discharge in the subthalamic nucleus of patients with Parkinson's disease. Exp Neurol 194:212-220. [Crossref]

7. Buzsaki G (2006) Rhythms of the Brain. New York: Oxford University Press.

8. Wiesel TN, Hubel DH (1963) Single cell responses in striate cortex of kittens deprived of vision in one eye. J Neurophysiol 26:1003-1017. [Crossref]

9. Singer W, Gray CM (1995) Visual feature integration and the temporal correlation hypothesis. Annu Rev Neurosci 18:555-586. [Crossref]

10. Carlson JD, Cleary DR, Cetas JS, Heinricher MM, Burchiel KJ (2010) Deep brain stimulation does not silence neurons in subthalamic nucleus in Parkinson's patients. $J$ Neurophysiol 103: 962-967. [Crossref]

11. Popovych OV, Lysyansky B, Rosemblum M, Pikovsky A, Tass PA (2017) Pulsatile desynchronizing delayed feedback for closed-loop deep brain stimulation. PLoS One 12:e0173363. [Crossref]

12. Brittain JS, Brown P (2014) Oscillations and the basal ganglia: motor control and beyond. Neuroimage 85 Pt 2: 637-647. [Crossref]

13. Lowet E, Roberts MJ, Bonizzi P, Karel J, and De Weerd P (2015) Quantifying neural oscillatory synchronization: a comparison between spectral coherence and phaselocking value approaches. PLoS One 11(1):e0146443. [Crossref]

14. Ko TW, Ermentrout GB (2007) Effects of axonal time delay on synchronization and wave formation in sparsely coupled neuronal oscillators. Phys Rev E Stat Nonlin Soft Matter Phys 76: 056206. [Crossref]

15. Little S, Brown P (2014) The functional role of beta oscillations in Parkinson's disease. Parkinsonism Relat Disord. 20S1:S44-S48. [Crossref]

16. Schoner G (2009). Development as change of system dynamics: stability, instability, and emergence. In Toward a Unified Theory of Development. Spencer J, Thomas MSC, McClelland JL (eds) Oxford: Oxford University Press.

17. Ermentrout GB, Beverlin B 2nd, Troyer T, Netoff TI (2011) The variance of phaseresetting curves. J Comput Neurosci 31: 185-197. [Crossref]
18. Ahn S, Rubchinsky LL (2013) Short desynchronization episodes prevail in synchronous dynamics of human brain rhythms. Chaos 23:1-8. [Crossref]

19. Ermentrout GB, Kopell N (1990). Oscillator death in systems of coupled neural oscillators. SIAM J Applied Math 50:125-146.

20. Deco G, Jirsa V, Friston KJ (2013) The dynamical and structural basis of brain activity. In Principles of Brain Dynamics: Global State Interactions. Rabinovich MI, Friston KJ, Varona P (eds). Cambridge, MA: MIT Press.

21. Ahn S, Rubchinsky LL (2017) Potential mechanisms and functions in intermittent neural synchronization. Front Comput Neurosci 11: 44. [Crossref]

22. Bridi JC, Hirth F (2018) Mechanisms of Îą-Synuclein Induced Synaptopathy in Parkinson's Disease. Front Neurosci 12: 80. [Crossref]

23. Baker SN, Olivier E, Lemon RN (1997) Coherent oscillations in monkey motor cortex and hand muscle EMG show task-dependent modulation. J Physiol 501:225-241. [Crossref]

24. Hammond C, Bergman H, Brown P (2007) Pathologial synchronization in Parkinson's disease: networks, models, and treatments. Trends Neurosci 30:357-364. [Crossref]

25. Compte A, Sanchez-Vives MV, McCormick DA, Wang XJ (2003) Cellular and network mechanisms of slow oscillatory activity $(>1 \mathrm{~Hz})$ and wave propagations in a cortical network model. J Neurophysiol 89: 2707-2725. [Crossref]

26. Timmermann L, Florin E (2012) Parkinson's disease and pathologial oscillatory activity: Is the beta band the bad guy? New lessons learned from low-frequency deep brain stimulation. Exp Neurol 233:123-125. [Crossref]

27. Deniau JM, Degos B, Bosch C, Maurice N (2010) Deep brain stimulation mechanisms beyond the concept of local functional inhibition. Eur J Neurosci 32:1080-1091. [Crossref]

28. Garcia L, D'Alessandro G, Bioulac B, Hammond C (2005) High-frequency stimulation in Parkinson's disease: more or less? Trends Neurosci 28: 209-216. [Crossref]

29. Windels F, Bruet N, Poupard A, Feuerstein C, Bertrand A, et al. (2003) Influence of the frequency parameter on extracellular glutamate and gamma-aminobutyric acid in substantia nigra and globus pallidus during electrical stimulation of subthalamic nucleus in rats. J Neurosci Res 15: 259-67. [Crossref]

30. Hernandez LF, Kubota Y, Hu D, Howe MW Lemaire N, et al. (2013) Selective effects of dopamine depletion and L-DOPA therapy on learning-related firing dynamics of striatal neurons. J Neurosci 33:4782-4795. [Crossref]

31. MacDonald AA, Seergobin KN, Owen AM, Tamjeedi R, Monchi O, et al (2013) Differential effects of Parkinson's Disease and dopamine replacement on memory encoding and retrieval. PLoS One 8(9):e74044-74053. [Crossref]

Copyright: $\odot 2018$ Larrivee D. This is an open-access article distributed under the terms of the Creative Commons Attribution License, which permits unrestricted use, distribution, and reproduction in any medium, provided the original author and source are credited. 